\title{
Decrease in number of genital chlamydia cases in Norrbotten, Sweden, October - November 2009: an indirect effect of pandemic influenza $\mathrm{A}(\mathrm{H} 1 \mathrm{~N} 1)$ ?
}

A Österlund (anders.osterlund@nll.se)1

1. Department for Communicable Disease Prevention and Control, County of Norrbotten, Sweden

Citation style for this article:

Österlund A. Decrease in number of genital chlamydia cases in Norrbotten, Sweden, October - November 2009: an indirect effect of pandemic influenza A(H1N1)? Euro Surveill. 2010;15(39):pii=19675. Available online: http://www.eurosurveillance.org/ViewArticle.aspx?Articleld=19675

Article published on 30 September 2010

A sudden reduction in the number of reported genital chlamydia cases was observed in Norrbotten County, Sweden, during October to November 2009. After exploring other possibilities, such as a reduction in the number of chlamydia tests analysed or a new Chlamydia trachomatis variant that had been undetected in standard laboratory tests, it was found likely that the decrease was an indirect effect of the 2009 influenza $A\left(\mathrm{H}_{1} \mathrm{~N}_{1}\right)$ pandemic due to reduced social interactions among young adults.

\section{Background}

Genital chlamydia infection has been a mandatorily notifiable disease in Sweden since 1988 [1]. Contact tracing is also mandatory for every case [1]. Since October 2009, the number of reported cases of genital chlamydia has decreased dramatically in Norrbotten, the northernmost Swedish county, which has a sparse population of 250,000 inhabitants. When comparing the number of monthly reported cases of genital Chlamydia trachomatis infections during the period January 2008 to October 2009 with that from November 2009 to July 2010, a $37 \%$ reduction was seen. This represents a fall in the monthly incidence from 33 per 100,000 population to 21 per 100,000 population. We have therefore investigated the possible causes of this sudden decrease.

\section{Number of genital chlamydia tests conducted}

One possible explanation for the decrease in the number of reported genital chlamydia cases is that there had been a reduction in the number of people tested for C. trachomatis. All C. trachomatis samples in the county are analysed at the microbiology laboratory at Sunderby Hospital in Luleå, Sweden. When comparing the mean number of monthly $C$. trachomatis tests at the laboratory during the period January 2008 to October 2009 with that from November 2009 to July 2010 , there had been only a $13 \%$ reduction (Figure 1 ), from 1,004 to 877 tests. The reason for this slight drop is not known. During August to September 2010, the number of tests increased again, and reached the level seen before October 2009. As the number of genital chlamydia cases fell by $37 \%$ during November 2009 to July 2010, however, it would seem that a reduction in testing was not the main explanation for the fall in the number of reported cases. As seen in Figure 1, by September 2010 the number of reported cases of genital chlamydia had reached the level seen before October 2009.

\section{Testing for a new variant of $C$. trachomatis}

The spread of a new variant of $C$. trachomatis in Sweden, which had been initially undetected due to a deletion in the cryptic plasmid, has been described previously $[2,3]$. In order to exclude the presence of a new, undetected variant, urine samples from 165 consecutive cases during March to April 2010 that were found to be $C$. trachomatis negative by real-time DNA amplification assay, using ProbeTec ET (Becton Dickinson), were analysed for the $C$. trachomatis ompA gene at the Section of Clinical Bacteriology, Department of Medical Sciences, Uppsala University, Sweden, using a modified method previously described [4] with other outer primers [5]. If a previously unknown variant had caused the decrease, at least five additional cases would have been found by this method. However, all samples were negative. We therefore consider it unlikely that there has been a new $C$. trachomatis variant circulating that has not been detected in standard laboratory tests.

\section{Number of sexual partners among persons with genital chlamydia}

In Norrbotten County, the number of sexual partners found per laboratory-confirmed case of genital chlamydia is used to monitor the quality of contact tracing. The number of sexual partners of confirmed cases, determined through contact tracing, was not seen to decrease from the period January 2008 to October 2009 to that from November 2009 to September 2010 (Figure 2). Although contact tracing might not reveal the true number of partners, it seems unlikely that a sudden decrease in the actual number of partners would have passed unnoticed with this method. Hence, we consider that a sudden decrease in the number of 


\section{FIGURE 1}

Reported cases of genital chlamydia and samples analysed for Chlamydia trachomatis (two-week moving averages), Norrbotten County, Sweden, by month, January 2008 - September 2010

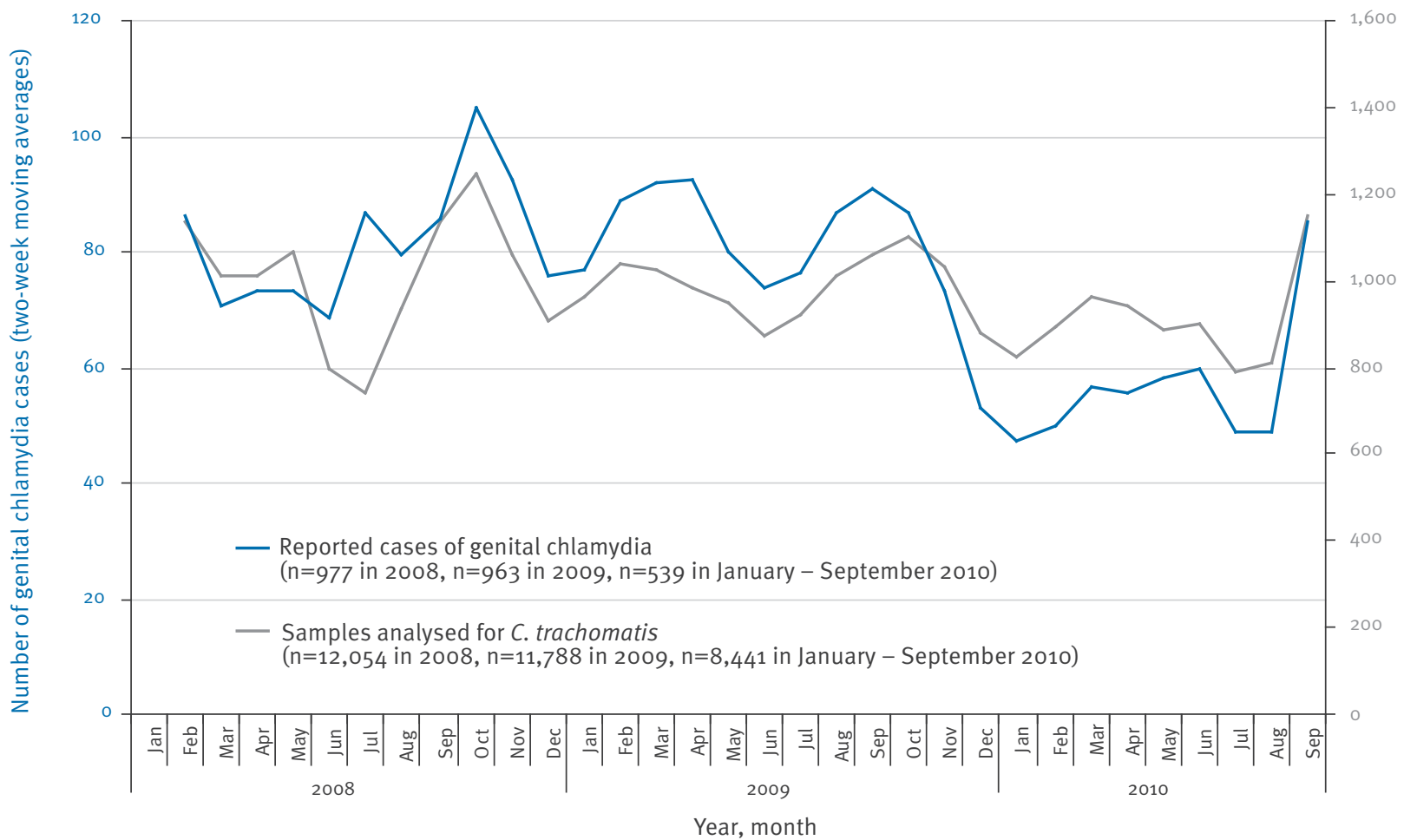

\section{FIGURE 2}

Reported cases of genital chlamydia and sexual partners per case determined by contact tracing (two-week moving averages), Norrbotten County, Sweden, by month, January 2008 - September 2010

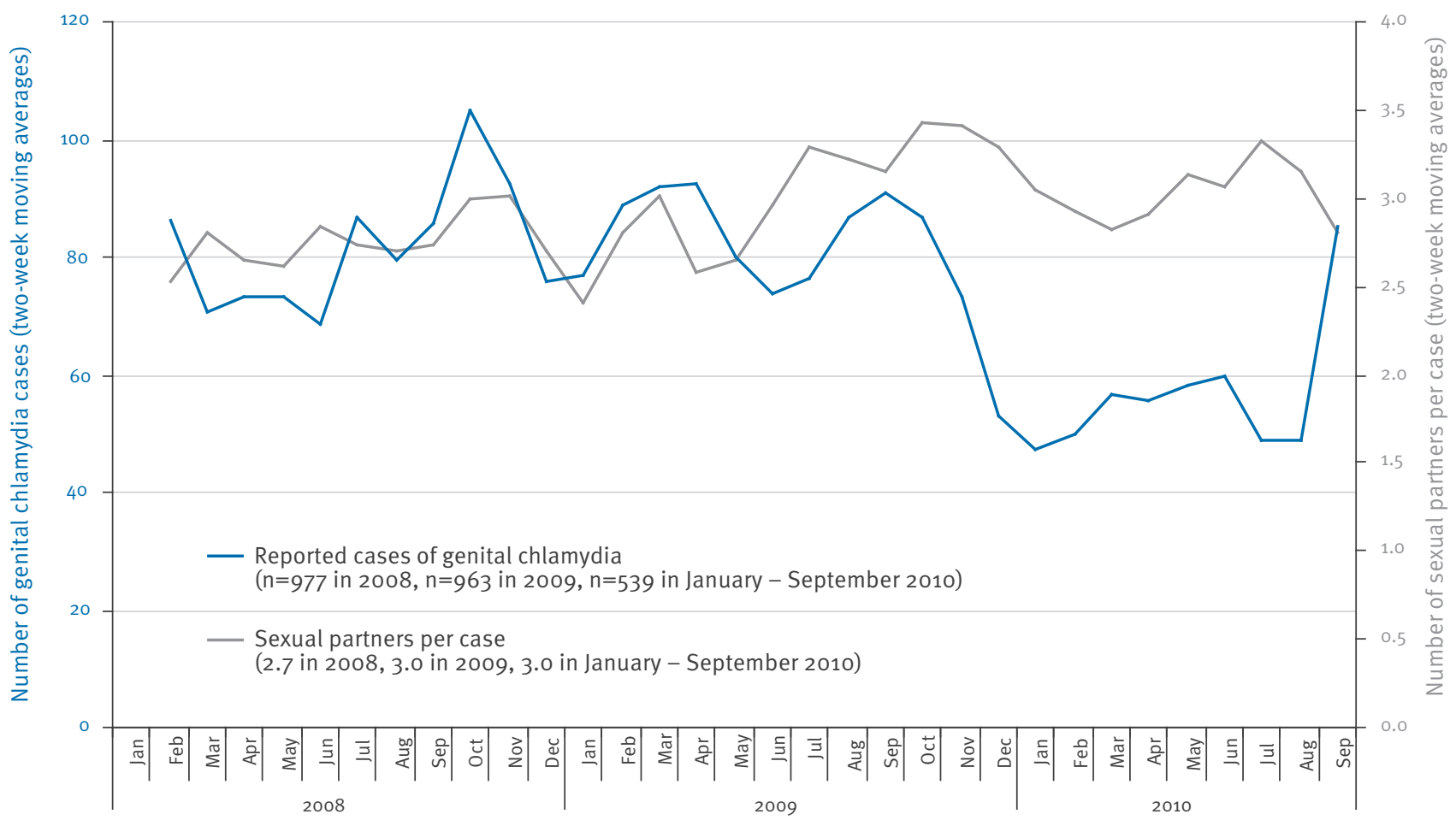

Year, month 
sexual partners among persons with confirmed genital chlamydia is not a plausible explanation for the observed decrease in the number of reported cases of C. trachomatis infection.

\section{Effect of pandemic influenza on social interactions among young adults}

Assuming that social interactions at nightclubs are related to sexual interactions, and thus may affect the spread of genital chlamydia, we also analysed whether the 2009 pandemic influenza $A\left(\mathrm{H}_{1} \mathrm{~N}_{1}\right)$ might have hampered nightclub visits among young adults. We chose to study the attendance of persons aged 18 to 25 years at the largest nightclub in the major city of the county as a representative of such social interactions. The nightclub usually opens in late September and closes at the end of May. During October to December 2009, the nightclub registered $15 \%$ fewer visitors compared with the same period the previous year. During the period January to May 2010, the number of visitors increased to a level exceeding that of the corresponding period in 2009 by $28 \%$. There is a clear concurrence between the influenza $A\left(\mathrm{H}_{1} \mathrm{~N}_{1}\right)$ pandemic in the region, the reduction in the number of nightclub visitors, and the dramatic decrease in number of reported genital chlamydia cases (Figure 3). Considering the increase in the number of nightclub visitors during February to May 2010, the monthly number of reported genital chlamydia cases would be expected to increase accordingly. As seen in Figure 3, by September 2010 the number of reported genital chlamydia cases has climbed to the level seen before October 2009 and the pandemic influenza $A\left(\mathrm{H}_{1} \mathrm{~N}_{1}\right)$ in the region.

In summary, the sudden decline in reported genital chlamydia cases in Norrbotten County, Sweden, from October to November 2009 was unlikely to be explained by fewer persons being tested or by a new C. trachomatis variant that had escaped detection. No new prevention programmes for sexually transmitted infections were introduced in the county after 2008, and it seems unlikely that earlier interventions could explain the sudden decline. However, there is an obvious coincidence in time between the regional occurrence of the 2009 pandemic influenza $A\left(\mathrm{H}_{1} \mathrm{~N}_{1}\right)$, the reduction of social interactions among young adults, and the dramatic decrease in the number of reported genital chlamydia cases. Thus, it seems likely that this reduction in the number of reported genital chlamydia cases was an indirect effect of the influenza pandemic. This is supported by the fact that in September 2010 the number of reported genital chlamydia cases had

\section{FIGURE 3}

Reported cases of genital chlamydia (two-week moving averages), number of reported 2009 pandemic influenza A(H1N1) cases, and percentage difference in number of nightclub visitors, Norrbotten County, Sweden, by month, January 2008 September 2010

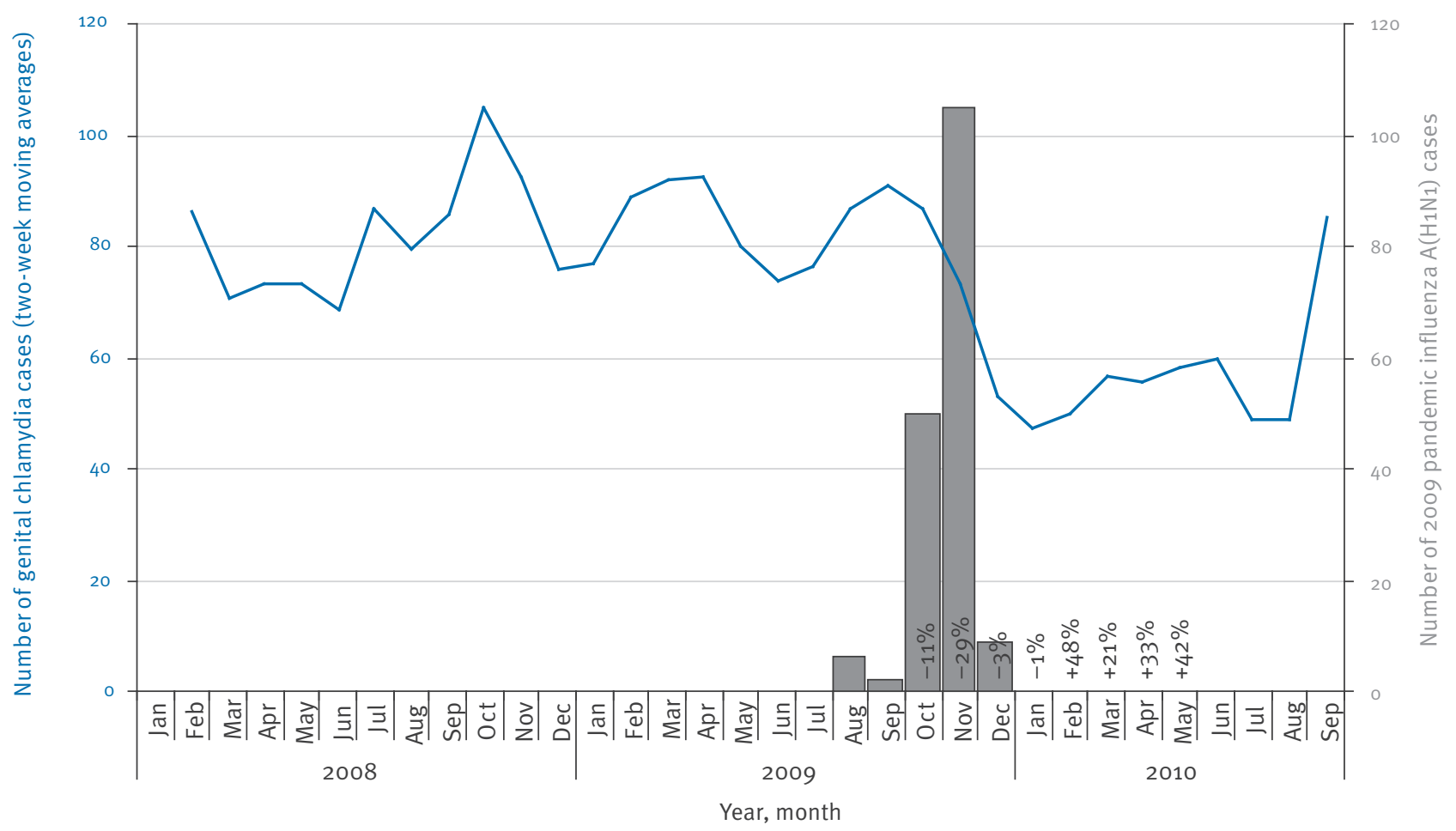

\footnotetext{
- Reported cases of genital chlamydia ( $n=977$ in 2008, $n=963$ in 2009, $n=539$ in January - September 2010)

$\square$ Reported cases of 2009 pandemic influenza $\mathrm{A}\left(\mathrm{H}_{1} \mathrm{~N}_{1}\right)(\mathrm{n}=172)$
}

Percentages in black show the monthly recorded increase or decrease in the number of nightclub visitors compared with the numbers seen in the same month the previous year. 
climbed to the level seen before October 2009 when the influenza pandemic occurred in the region.

\section{References}

1. [The Communicable Disease Act] (1988:1472). Stockholm: Ministry of Health \& Social Affairs; 1989 . Swedish. Available from: http://www.notisum.se/rnp/sls/lag/19881472.HTM

2. Ripa T, Nilsson P. A variant of Chlamydia trachomatis with deletion in cryptic plasmid: implications for use of PCR diagnostic tests. Euro Surveill. 2006;11(45). pii=3076. Available from: http://www.eurosurveillance.org/ViewArticle. aspx?Articleld $=3076$

3. Klint M, Hadad R, Christerson L, Loré B, Anagrius C, Österlund $A$, et al. Prevalence trends in Sweden for the new variant of Chlamydia trachomatis. Clin Microbiol Infect. 2010 Jul 15. [Epub ahead of print]

4. Lysen M, Österlund A, Rubin CJ, Persson T, Persson I, Herrmann B. Characterization of ompA genotypes by sequence analysis of DNA from all detected cases of Chlamydia trachomatis infections during 1 year of contact tracing in a Swedish County. J Clin Microbiol. 2004;42(4):1641-7.

5. Harding-Esch EM, Christerson L, Grannas K, Roberts $\mathrm{CH}$, Holland MJ, Andreasen AA, et al. Multi-locus sequence typing: a useful tool for trachoma molecular epidemiology. In: Stary A, Byrne GI, Caldwell H, et al., editors. Chlamydial infections. Proceedings of the 12th International Symposium on Human Chlamydial Infections; Salzburg 2010 Jun 20-25. 